\title{
ON A PROBLEM OF LOHWATER ABOUT THE ASYMPTOTIC BEHAVIOUR OF NEVANLINNA'S CLASS
}

\author{
J. S. HWANG ${ }^{1}$
}

\begin{abstract}
Let $f(z)$ be meromorphic in $|z|<1$ and let the radial limits $\lim _{r \rightarrow 1} f\left(r e^{i \theta}\right)$ exist and have modulus 1 for almost all $e^{i \theta} \in A=\left\{e^{i \theta}: \theta_{1}<\theta<\right.$ $\left.\theta_{2}\right\}$. If $P$ is a singular point of $f(z)$ on $A$, then every value of modulus 1 which is not in the range of $f(z)$ at $P$ is an asymptotic value of $f(z)$ at some point of each subarc of $\boldsymbol{A}$ containing the point $\boldsymbol{P}$. This answers in the affirmative sense a question of $\mathbf{A}$. J. Lohwater.
\end{abstract}

1. Introduction. In 1953 [2, Theorem 3], Lohwater proved the following result: Let $f(z)$ be meromorphic in the unit disk $D=\{z:|z|<1\}$ with bounded characteristic in the sense of Nevanlinna, and let the radial $\operatorname{limits}_{\lim _{r \rightarrow 1}} f\left(r e^{i \theta}\right)=f\left(e^{i \theta}\right)$ have modulus 1 for almost all $e^{i \theta} \in A=\left\{e^{i \theta}: \theta_{1}<\theta<\theta_{2}\right\}$. If $P$ is a singular point of $f(z)$ on $A$, then every value of modulus 1 which is not in the range of $f(z)$ at $P$ is an asymptotic value of $f(z)$ at some point of each subarc of $A$ containing the point $P$. He then asked as to whether this result is still true if $f(z)$ is not of bounded characteristic (see [2, p. 156]). The following theorem answers this question in the affirmative sense.

THEOREM 1. Let $f(z)$ be meromorphic in $D$ and let the radial limits $\lim _{r \rightarrow 1} f\left(r e^{i \theta}\right)=$ $f\left(e^{i \theta}\right)$ exist and have the modulus 1 for almost all $e^{i \theta} \in A=\left\{e^{i \theta}: \theta_{1}<\theta<\theta_{2}\right\}$. If $P$ is a singular point of $f(z)$ on $A$, then every value of modulus 1 which is not in the range of $f(z)$ at $P$ is an asymptotic value of $f(z)$ at some point of each subarc of $A$ containing the point $P$.

2. Maximum principle. To prove Theorem 1, we shall need the following strong form of maximum principle due to Collingwood and Lohwater [1, Theorem 5.3].

Lemma 1. If $f(z)$ is analytic and bounded, $|f(z)|<M$, in $D$, and if the radial limit values $f\left(e^{i \theta}\right)$ are in modulus not greater than $m<M$ almost everwhere on $|z|=1$, then $|f(z)|<m$ everywhere in $D$, unless $f(z)$ is a constant of modulus $m$.

3. Proof of Theorem 1. Let $Q$ be a value of modulus 1 which is not in the range of $f(z)$ at $P$ and let $g(z)$ be the function defined by $g(z)=1 /(f(z)-Q)$, for $z \in D$. Then there is a number $\delta>0$ such that the function $g(z)$ is analytic in the set $D_{\delta}(P)=D \cap\{z:|z-P|<\delta\}$.

There are two cases to be considered: either the function $g(z)$ is bounded in the domain $D_{\delta}(P)$ or it is not. In the first case, we let $z=z(w)$ be a conformal

Received by the editors March 25, 1980 and, in revised form, July 29, 1980.

AMS (MOS) subject classifications (1970). Primary 30A72; Secondary 30A76.

Key words and phrases. Asymptotic behaviour, Nevanlinna's class.

${ }^{1}$ I am indebted to the referee for his many valuable comments and corrections in this research. 
mapping from the unit disk $D_{w}=\{w:|w|<1\}$ onto the domain $D_{\delta}(P)$. Then by the Nevanlinna theory, see [1, p. 39], the functions $g(z(w))$ and $f(z(w))$ are both of bounded characteristic in $D_{w}$. In this case, the result follows from Lohwater's theorem [2, Theorem 3]. We may, therefore, assume that the function $g(z)$ is unbounded in $D_{\delta}(P)$.

For convenience, we write $P=e^{i \alpha}$, where $\theta_{1}<\alpha<\theta_{2}$. From the hypothesis, we can choose two numbers $\alpha_{1}$ and $\alpha_{2}$ with $\theta_{1}<\alpha_{1}<\alpha<\alpha_{2}<\theta_{2}$ and $\alpha_{2}-\alpha_{1}<\delta$ such that the radial $\operatorname{limits} \lim _{r \rightarrow 1} f\left(r e^{i \alpha_{j}}\right)=f\left(e^{i \alpha_{j}}\right)$ exist for $j=1,2$. This in turn implies the following radial limits exist

$$
\lim _{r \rightarrow 1} g\left(r e^{i \alpha_{j}}\right)=1 /\left(f\left(e^{i \alpha_{j}}\right)-Q\right)=g\left(e^{i \alpha_{j}}\right), \quad \text { for } j=1,2 .
$$

Let $R_{j}$ be the radius ending at $e^{i \alpha_{j}}$ and let $S_{j}$ be the point of intersection of $R_{j}$ with the boundary of $D_{\delta}(P)$ which lies within the disk $D$, where $j=1,2$. Denote by $T$ the portion of the boundary of $D_{\delta}(P)$ between $S_{1}$ and $S_{2}$. If $f(z)$ has the radial limit $Q$ on either $R_{1}$ or $R_{2}$, then there is nothing more to prove, so we may suppose that the radial limits of $f(z)$ along $R_{1}$ and $R_{2}$ are both different from $Q$. Then by (1) we find that the function $g(z)$ is bounded on the union $R_{1} \cup T \cup R_{2}$, so that for some $M>0$,

$$
|g(z)|<M, \text { for } z \in R_{1} \cup T \cup R_{2} \text {. }
$$

Let $G$ be the domain bounded by $R_{1} \cup T \cup R_{2}$ and the $\operatorname{arc} A\left(\alpha_{1}, \alpha_{2}\right)=\left\{e^{i \theta}\right.$ : $\left.\alpha_{1}<\theta<\alpha_{2}\right\}$. If the function $g(z)$ is bounded in $G$, then by the same argument as before we know that the assertion follows from Lohwater's theorem. We may, therefore, assume that this function $g(z)$ is unbounded in $G$. Let $M_{1}>M$ and let $H=\left\{z:|z|<1\right.$ and $\left.|g(z)|>M_{1}\right\}$ and $H_{1}=H \cap G$. Since $g(z)$ is unbounded in $G$, the domain $H_{1}$ is not empty. Moreover, from (2) we can see that the boundary of $H_{1}$ is disjoint from the set $R_{1} \cup T \cup R_{2}$. Let $H_{1}^{*}$ be a component of $H_{1}$. We shall prove that $g(z)$ is unbounded in $H_{1}^{*}$. Suppose on the contrary that $|g(z)|<N$, for $z \in H_{1}^{*}$, where $N>M_{1}$. As before, let $z=z(w)$ be a conformal mapping from $D_{w}$ onto $H_{1}^{*}$. For almost all $\theta \in[0,2 \pi]$, either $\lim _{r \rightarrow 1}\left|g\left(z\left(r e^{i \theta}\right)\right)\right|=M_{1}$ or $\lim _{r \rightarrow 1} g\left(z\left(r e^{i \theta}\right)\right)$ lies on the line $L$ which is the image of the circle $|z|=1$ under the mapping $\phi(z)=1 /(z-Q)$. The line $L$ meets the circle $|z|=M_{1}$ from elementary considerations. Since $g(z(w))$ is a nonconstant bounded function on $D_{w}$, if we set $W=g\left(z\left(D_{w}\right)\right)$ then there exist points $\zeta_{1}$ and $\zeta_{2}$ such that $\zeta_{1} \in W, \zeta_{2}$ is not in the closure of $W$, and the distance from $\zeta_{1}$ to $\zeta_{2}$ is less than half the distance from $\zeta_{2}$ to $L \cup\left\{z:|z|=M_{1}\right\}$. Let $h(w)=1 /\left(g(z(w))-\zeta_{2}\right)$. Then $h(w)$ is a bounded analytic function on $D_{w}$ which assumes the value $1 /\left(\zeta_{1}-\zeta_{2}\right)$, but $h(w)$ has radial limits of modulus at most $1 /\left(2\left|\zeta_{1}-\zeta_{2}\right|\right)$ almost everywhere on $|w|=1$, in violation of Lemma 1. It follows that $g(z)$ is unbounded on $H_{1}^{*}$. Based on this property, we shall construct a path $\Gamma$ such that $\Gamma \subset G$ and $g(z)$ tends to infinity along this path $\Gamma$.

We begin by choosing $z_{1} \in H_{1}^{*}$. Let $r_{2}$ and $M_{2}$ be two positive numbers such that $\left|z_{1}\right|<r_{2}<1$ and

$$
M_{1}>\max |g(z)|, \quad \text { for } z \in H_{1}^{*} \cap\left\{z:|z|<r_{2}\right\} .
$$


Choose $H_{2}^{*}$ a component of the domain $H_{2}=\left\{z: z \in H_{1}^{*}\right.$ and $\left.|g(z)|>M_{2}\right\}$. Then $H_{2}^{*}$ is not empty because $g(z)$ is unbounded in $H_{1}^{*}$. Choose a point $z_{2} \in H_{2}^{*}$ and join $z_{1}$ to $z_{2}$ by an arc $\Gamma_{1}$ lying in $H_{1}^{*}$. Then clearly we have $|g(z)|>M_{1}$, for $z \in \Gamma_{1}$, where $\Gamma_{1} \subset H_{1}^{*}$. Continuing this process, we can construct four sequences $\left\{H_{n}^{*}\right\},\left\{z_{n}\right\},\left\{\Gamma_{n}\right\}$, and $\left\{M_{n}\right\}$ such that

$H_{1}^{*} \supset H_{2}^{*} \supset \cdots, \Gamma_{n} \subset H_{n}^{*}$,

$\Gamma_{n}$ joins $z_{n}$ to $z_{n+1},\left|z_{n}\right| \uparrow 1$, as $n \rightarrow \infty$, and

$|g(z)|>M_{n}$, for $z \in \Gamma_{n}$, where $M_{n} \uparrow \infty$, as $n \rightarrow \infty$.

Letting $\Gamma=\cup \Gamma_{n}$, we have that $\Gamma \subset G$ and that $g(z) \rightarrow \infty$ as $|z| \rightarrow 1$ along $\Gamma$. It then follows that the function $f(z)$ tends to the value $Q$ along the path $\Gamma$.

By our construction, $\Gamma_{A}=\bar{\Gamma} \cap\{z:|z|=1\} \subset \overline{A\left(\alpha_{1}, \alpha_{2}\right)}$ (where we use $\bar{E}$ to denote the closure of the set $E$ ). If $\Gamma_{A}$ is a single point, then $f$ has the asymptotic value $Q$ at this point. But if $\Gamma_{A}$ contains two points, then $\Gamma_{A}$ is a closed subarc of $\overline{A\left(\alpha_{1}, \alpha_{2}\right)}$, and so the radial limit of $f$ at almost every point of $\Gamma_{A}$ must be $Q$, since each radius to a point of the interior of the $\operatorname{arc} \Gamma_{A}$ must meet the $\operatorname{arc} \Gamma$ at points arbitrarily close to $|z|=1$. This completes the proof.

Finally, we remark that the hypotheses of Theorem 1 are insufficient to ensure that the function $f$ is of bounded characteristic. Lohwater [2, p. 155] has constructed a function of unbounded characteristic having radial limits of modulus 1 at almost all points of the unit circle.

\section{REFERENCES}

1. E. F. Collingwood and A. J. Lohwater, The theory of cluster sets, Cambridge Univ. Press, London and New York, 1966.

2. A. J. Lohwater, On the Schwarz reflection principle, Michigan Math. J. 2 (1953-1954), 151-156.

Instrtute of Mathematics, Academia Sinica, Taipei, Tatwan, Repubuic of China 